\title{
Dual immunosuppression enhances vasomotor injury: Interactive effect between endothelin-1 and nitric oxide bioavailability
}

Danny Ramzy, MD, Laura C. Tumiati, BSc, Elissa Tepperman, BSc, Rohit Sheshgiri, BSc, Jessica Jackman, BSc, Mitesh Badiwala, MD, and Vivek Rao, MD, PhD

From the Heart Transplant Program, Peter Munk Cardiac Center, Toronto General Hospital; Division of Cardiac Surgery, University of Toronto; and Division of Cardiology, University of Toronto, Toronto, Ontario, Canada

Supported by the Heart and Stroke Foundation of Ontario (Grant NA 5868), Canadian Institutes for Health Research, Thoracic Surgery Foundation for Research and Education, Tailored Advanced Collaborative Training in Cardiovascular Science, and Physician Services Incorporated Foundation Grant for Research Fellows. Dr Ramzy is a Research Fellow of the Thoracic Surgery Foundation for Research and Education, Policy Studies Institute, and Tailored Advanced Collaborative Training in Cardiovascular Sciences. Dr Rao is a Canadian Institutes of Health Research New Investigator.

Manuscript accepted for the C. Walton Lillehei Resident Forum Session at the Annual Meeting of the American Association for Thoracic Surgery.

Read at the Eighty-seventh Annual Meeting of The American Association for Thoracic Surgery, Washington, DC, May 5-9, 2007.

Received for publication May 14, 2007; revisions received Aug 18, 2007; accepted for publication Sept 6, 2007.

Address for reprints: Vivek Rao, MD, PhD, Toronto General Hospital, Division of Cardiovascular Surgery, 4N-464, 200 Elizabeth Street, Toronto, Ontario, Canada M5G 2C4 (E-mail: vivek.rao@uhn.on.ca).

J Thorac Cardiovasc Surg 2008;135:938-44 $0022-5223 / \$ 34.00$

Copyright $(\subset 2008$ by The American Association for Thoracic Surgery

doi:10.1016/j.jtcvs.2007.09.075
Objective: Cyclosporine A and corticosteroids are associated with many side effects, such as endothelial dysfunction and transplant vasculopathy. We examined the effects of cyclosporine A and hydrocortisone exposure on endothelial function of the rat thoracic aorta.

Methods: Lewis rats were injected with cyclosporine A, hydrocortisone, cyclosporine A + hydrocortisone, or intraperitoneal saline daily for 2 weeks. Endothelialdependent and independent vascular relaxation were assessed in isolated segments of thoracic aorta, as well as endothelin-1-induced vasoreactivity. Protein expression of endothelial nitric oxide synthase, endothelin $\mathrm{A}_{\mathrm{A}}$, and endothelin $\mathrm{B}_{\mathrm{B}}$ receptors were also determined in the thoracic aorta.

Results: Exposure to cyclosporine A and cyclosporine A + hydrocortisone resulted in a reduction in endothelial-dependent vasorelaxation compared with control and hydrocortisone $(P=.001)$. Cyclosporine $\mathrm{A}$ and hydrocortisone-treated rats demonstrated increased vasoreactivity to endothelin-1 compared with control, whereas cyclosporine $\mathrm{A}+$ hydrocortisone treatment resulted in a synergistic increase $(P=.04)$. All treatment groups displayed a significant reduction in endothelial nitric oxide synthase expression compared with control $(P=.001)$. Endothelin ${ }_{\mathrm{A}}$ receptor expression was increased in all treatment groups with a synergistic effect seen after cyclosporine A + hydrocortisone treatment. No differences were seen in endothelin $n_{B}$ receptor expression.

Conclusion: Cyclosporine A and hydrocortisone induce vasomotor dysfunction with a synergistic impairment observed after concomitant exposure. Our findings suggest that the resultant vasomotor dysfunction is the result of alterations in both nitric oxide and endothelin-1 regulation.

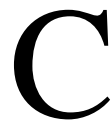
yclosporine $\mathrm{A}(\mathrm{CyA})$ was the first antirejection drug that affected the results of clinical heart transplantation by reducing the incidence and severity of rejection and remains an important component of modern immunosuppressive therapy. Unfortunately, CyA is associated with many negative side effects, such as nephrotoxicity, hepatotoxicity, neurotoxicity, and hypertension. ${ }^{1-3}$ Other adverse effects include endothelial dysfunction and the development of transplant vasculopathy. ${ }^{1,4-10}$ CyA can influence transplant vasculopathy by increased plasma lipid concentrations, causing hypertension, or by direct injury to the endothelium. ${ }^{10-13} \mathrm{It}$ is believed that the endothelial injury induced by CyA results in impaired vascular homeostasis and transplant coronary disease.

The mechanisms by which CyA results in endothelial dysfunction are not fully elucidated. However, CyA is known to impair vasodilation ${ }^{14-17}$ and may induce vasoconstriction. ${ }^{18-20}$ Potential mechanisms resulting in vasospasm include the increased release of vasoconstrictors or increased sensitivity to these vasoconstrictors. One such vasoconstrictor is endothelin (ET)-1. The effect of CyA on plasma levels of 


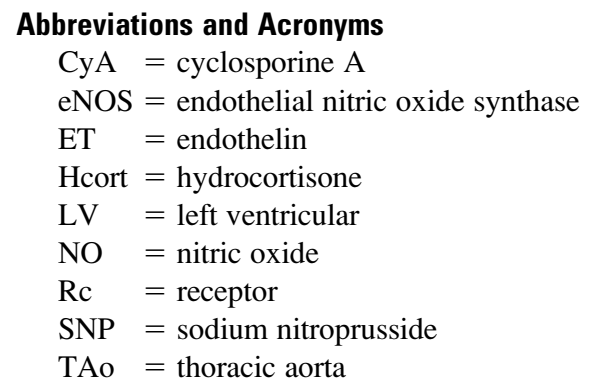

ET-1 remains controversial. Most investigators have found an increase in ET-1 levels after CyA treatment, although this is not a consistent finding. ${ }^{15,19,21-27}$ Furthermore, altered nitric oxide (NO) homeostasis may result in an impaired vasodilatory response. ${ }^{15}$ Impaired NO homeostasis may be a result of decreases in mRNA or protein expression of endothelial nitric oxide synthase (eNOS) in CyA-treated patients. Several investigators have demonstrated that eNOS RNA expression is increased after CyA treatment, ${ }^{28}$ suggesting that impaired NO production may be due to decreases in eNOS protein synthesis or a shift to free radical production. ${ }^{29}$ There is also evidence that CyA generates free radicals. ${ }^{4}$ These free radicals may result in direct endothelial injury and impaired vasomotor function.

Corticosteroids are another commonly used antirejection agent also associated with several side effects. Corticosteroids can inhibit the release of vasodilators, such as histamine and prostacyclin. We hypothesized that the combination of CyA and corticosteroids (commonly used after solid organ transplantation) results in a synergistic impairment of vascular function. Our investigations assessed the role of $\mathrm{CyA}$, and hydrocortisone (Hcort) on the development of endothelial dysfunction in a rodent model of vascular injury. Although these investigations have direct relevance to transplant vasculopathy, we intentionally avoided a transplant model to eliminate the confounding effects of immune-mediated injury. Thus, these studies evaluate the drug-specific changes to vascular function after sustained exposure. Specifically, we examined the effects of CyA and Hcort exposure on NO homeostasis and ET-1 signaling.

\section{Materials and Methods}

Animal care conformed to the "Canadian Council on Animal Care Guide to the Care and Use of Experimental Animals" (National Institutes of Health publication no 86-23, revised 1996).

Male Lewis rats $(\mathrm{n}=16)(200-300 \mathrm{~g})$ were administered the drug of interest (saline control, CyA $(5 \mathrm{mg} / \mathrm{kg}$ ) or Hcort $(20 \mathrm{mg}$ / $\mathrm{kg})$ ) via peritoneal injection daily for a period of 2 weeks before assessment of endothelial function. On the day of sacrifice, rats were anesthetized using isoflurane. Median sternotomy was then performed, the heart was excised for myocardial tissue sampling, and segments of aorta were procured for assessment of endothelial function. Before heart excision, $1 \mathrm{~mL}$ of blood from the right ventricle was collected for analysis of ET-1 plasma levels. The rats were then exsanguinated under general anesthesia.

\section{Endothelial Function Assessment}

Endothelial-dependent and independent vascular relaxation were assessed in isolated segments of thoracic aorta (TAo) after treatment. The TAo was dissected, and vascular segments $(5 \mathrm{~mm}$ in length) were used for the assessment of in vitro vascular function using a small vessel myograft for isometric tension recording. After mounting the vessel on a pressure transducer, maximum vasoconstriction was achieved with exposure to phenylephrine. After stabilization, endothelial-dependent relaxation was assessed by incremental exposure to acetylcholine. Endothelial-independent relaxation was assessed by incremental exposure to sodium nitroprusside (SNP). The maximum relaxation from phenylephrine-induced vasoconstriction $(\mathrm{Emax} \%)$ was compared between groups. $\mathrm{ED}_{50}$, calculated as the concentration required to achieve half-maximum vasorelaxation, was compared between groups. Sensitivity to vasospasm was assessed in vessels following stabilization after SNP washout. Incremental exposure to ET-1 was performed, and \% Cmax was calculated as the maximum increase in tension from baseline. Each animal yielded 2 aortic segments. Data were included if the variability between segments was less than $10 \%$, and data were averaged to yield 1 result per animal.

\section{Plasma Measurements}

Venous blood was aspirated from the right ventricle before exsanguination. CyA trough levels were performed on whole blood. For ET-1 levels, blood samples were centrifuged $(14,000 \mathrm{rpm})$ to collect the plasma fraction, which was snap-frozen in liquid nitrogen and stored at $-80^{\circ} \mathrm{C}$. ET-1 in plasma was extracted using $\mathrm{C}^{18}$ SepPack (Waters Corporation, Milford, Mass) columns after acidification with $1 \%$ trifluoroacetic acid. Plasma ET-1 levels were measured using a commercial enzyme-linked immunosorbent assay (Biomedica, Vienna, Austria).

\section{Assessment of Oxidative Injury}

8 -isoprostane levels were measured as an indicator of free radicalmediated injury. ${ }^{30} 8$-isoprostane is the stable end product of arachidonic acid oxidation generated by reactive oxygen species injury. ${ }^{31}$ Determination of 8-isoprostane levels in left ventricular (LV) myocardial tissue was performed using a commercially available kit (Cayman Chemical Company, Ann Arbor, Mich). The percentage from baseline (LV from animals that received no intraperitoneal injections) was calculated to compare differences between groups.

\section{Western Blot Analysis}

LV biopsies and the TAo were immediately collected after harvesting. Biopsy specimens were snap-frozen in liquid nitrogen and stored at $-80^{\circ} \mathrm{C}$ until analyzed. Biopsies were homogenized at $4^{\circ} \mathrm{C}$ and prepared for analysis. Protein determination was determined by the method described by Bradford. ${ }^{32}$

Western blot determined the protein expression of inducible NOS, eNOS, tumor necrosis factor- $\alpha$, and transforming growth factor- $\beta$ with the use of protein-specific monoclonal antibodies (BD Biosciences, Mississauga, Canada) and $\mathrm{ET}_{\mathrm{A}}$ and $\mathrm{ET}_{\mathrm{B}}$ receptors (Rc's) with the use of protein-specific polyclonal antibodies 
(Chemicon, Temecula, Calif). Samples were separated using 4\% stacking and $10 \%$ running tris-glycine sodium dodecylsulfate-polyacrylamide gel electrophoresis gels. Gels were then transferred to polyvinylidene difluoride membranes. Blocking was performed for 1 hour at room temperature using blocking buffer. The blots were exposed to the primary antibody for 12 hours at $4^{\circ} \mathrm{C}$ and then incubated with secondary antibody for 1 hour after washing. Comparisons between groups were performed using densitometric analysis. TAo densitometric data were corrected for $\beta$-actin expression, and LV data were corrected for glyceraldehyde-3-phosphate dehydrogenase expression. No differences in $\beta$-actin and glyceraldehyde-3-phosphate dehydrogenase protein expression were seen between groups at any time point.

\section{Statistical Analysis}

Statistical analysis was performed with the SAS statistical software program V8.2 (SAS institute, Inc, Cary, NC). Continuous data were analyzed by analysis of variance and are expressed as the mean \pm standard deviation. When the F-statistic of the analysis of variance was significant $(P<.05)$, a Duncan's multiple range test was performed to specify differences between groups.

\section{Results}

All animals survived until the day of sacrifice with no complications except for 1 rat with a gingival abscess in the Hcort group. There was minimal variability from aortic segments within each animal, and no animals were excluded from the study. CyA levels in both treatment groups were similar: CyA $(61.7 \pm 11 \mathrm{ng} / \mathrm{mL})$ and $\mathrm{CyA}+$ Hcort $(63.2 \pm 9 \mathrm{ng} / \mathrm{mL})$.

\section{Endothelial Function}

Endothelial-dependent vasorelaxation of thoracic aortic rings was impaired after CyA treatment compared with control (Figure 1, A). CyA and CyA + Hcort treatment resulted in an Emax $\%$ of $33 \% \pm 2 \%$ and $30 \% \pm 7 \%$, respectively, which was significantly $(P=.001)$ lower than that of control at $46 \% \pm 1 \%$. However, isolated Hcort treatment (Emax\%: $51 \% \pm 1 \%$ ) did not result in any significant impairment of endothelial-dependent vasorelaxation compared with control. Assessment of endothelial-independent Emax\% to SNP revealed no significant differences between groups $(P=.2)$ (Figure $1, B)$. However, when examining the concentration of SNP necessary to achieve $50 \%$ of maximal vasodilatory response, significant differences were seen between groups. CyA-treated $\left(\mathrm{ED}_{50} 3.2 \times 10^{-8} \pm 0.2\right.$ mole/L) and CyA + Hcort-treated $\left(\mathrm{ED}_{50} 4.0 \times 10^{-8} \pm 0.3\right.$ mole/L) animals required approximately double the concentration of SNP compared with both control $\left(\mathrm{ED}_{50} 1.6 \times 10^{-8}\right.$ $\pm 0.1 \mathrm{~mole} / \mathrm{L})$ and Hcort $\left(\mathrm{ED}_{50} 1.3 \times 10^{-8} \pm 0.1 \mathrm{~mole} / \mathrm{L}\right)$ $(P=.01)$. Differences between CyA + Hcort and CyA alone were also significant $(P<.05)$.

Significant differences were also found in vessel sensitivity to ET-1 exposure. Sensitivity to vasospasm (\% Cmax) was greater in CyA and Hcort-treated groups $(P=.04)$ compared with control (CyA: $256 \% \pm 11 \%$ vs Hcort: $238 \% \pm 7 \%$ vs

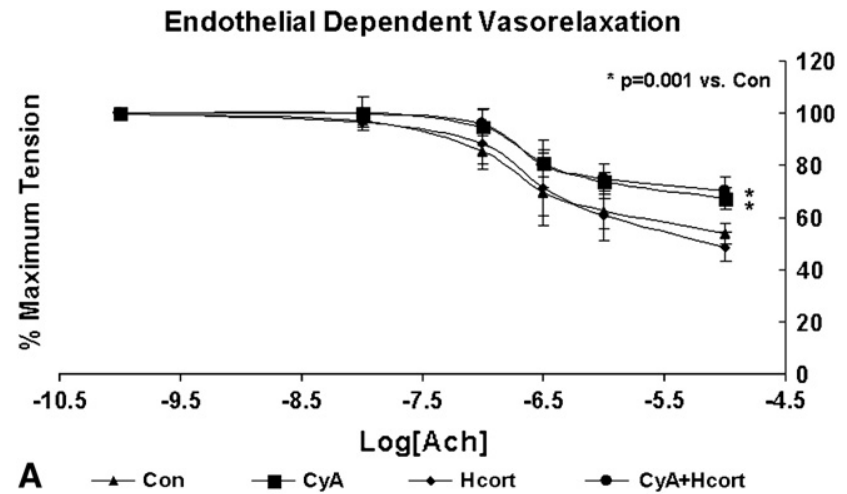

Endothelial Independent Vasorelaxation

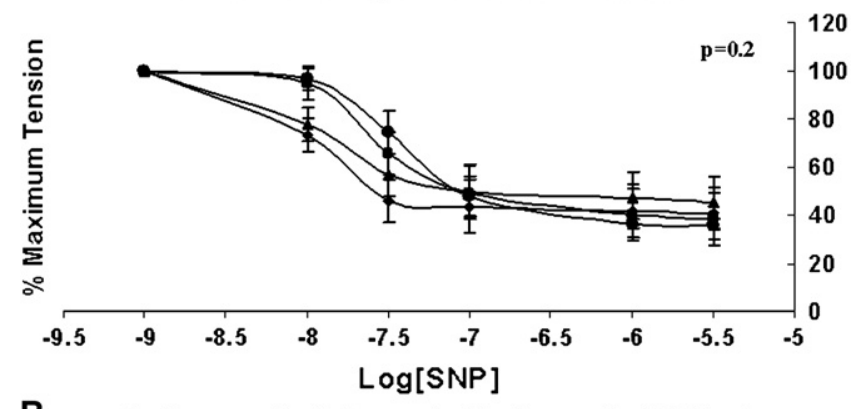

B $\rightarrow$ Con $\rightarrow$ - СуA $\rightarrow$ Hcort $\rightarrow$ CyA +Hcort

Sensitivity to Vasospasm

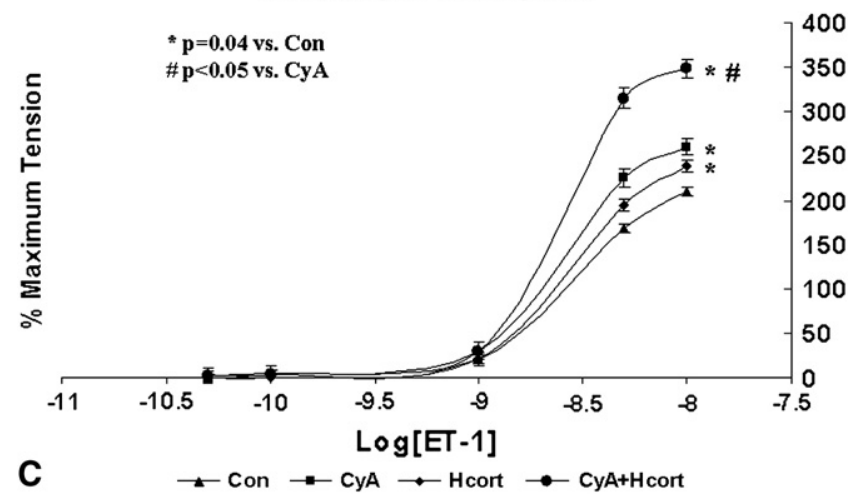

Figure 1. A, Endothelial-dependent vasodilation in rat TAo. The graph depicts the cumulative dose-response curves to acetylcholine in aortic segments. CyA and CyA + Hcort treatment results in impaired endothelial-dependent vasorelaxation compared with Hcort and control. B, Endothelial-independent vasodilation in rat TAo. The graph depicts the cumulative dose-response curves to SNP in aortic segments. No differences in \%Emax were seen between groups. C, Cumulative dose-response curves to ET-1 in aortic segments of treated rats. Both CyA and Hcort increase vasosensitivity to ET-1. Concomitant treatment synergistically increases vessel sensitivity to ET-1. CyA, Cyclosporine A; Hcort, hydrocortisone; ET, endothelin; Con, control; Ach, acetylcholine. 
control: $209 \% \pm 7 \%$ ) (Figure 1, C). Concomitant treatment with CyA + Hcort (\%Cmax: 348\% $\pm 10 \%$ ) resulted in a synergistic increase in ET-1 induced vasospasm compared with CyA and Hcort treatment alone.

\section{Plasma Endothelin-1 Levels}

Plasma ET-1 levels were not different between groups (CyA + Hcort: $1.0 . \pm 0.1 \mathrm{fmol} / \mathrm{L}$ vs CyA: $0.98 \pm 0.1 \mathrm{fmol} / \mathrm{L}$ vs Hcort: $0.97 \pm 0.1 \mathrm{f} \mathrm{mol} / \mathrm{L}$ vs control: $0.97 \pm 0.1 \mathrm{fmol} / \mathrm{L}$, $P=.05)$. Control animals had an ET-1 plasma concentration that is in the expected normal range. ${ }^{19}$

\section{Oxidative Injury}

$\mathrm{CyA}+$ Hcort and $\mathrm{CyA}$ treatment resulted in a greater increase in oxidative injury as measured by changes in 8-isoprostane levels compared with both control and Hcort groups (CyA + Hcort: $162 \% \pm 9 \%$ vs CyA: $150 \% \pm 6 \%$ vs Hcort: $113 \% \pm 2 \%$ vs control: $107 \% \pm 4 \%, P=.05)$.

\section{Endothelin Receptor Expression}

Thoracic aortic $\mathrm{ET}_{\mathrm{A}} \mathrm{Rc}$ protein expression was significantly $(P=.004)$ increased in all treatment groups compared with control with concomitant treatment, resulting in an additive increase in $\mathrm{ET}_{\mathrm{A}}$ Rc expression (Figure 2, $A$ and $B$ ). However, $\mathrm{ET}_{\mathrm{B}} \mathrm{Rc}$ protein expression was not different between groups $(P=.29)$.

\section{Nitric Oxide Synthase Expression}

eNOS protein expression was lower in treated animals (CyA and Hcort) compared with control $(P<.001)$ (Figure $2, C$ ). However, CyA + Hcort exposure resulted in a significant synergistic reduction in eNOS protein expression. However, inducible NOS expression was not induced in any of the experimental groups.

\section{Proinflammatory Cytokine Expression}

Myocardial protein expression of tumor necrosis factor- $\alpha$ and transforming growth factor- $\beta$ did not differ between groups.

\section{Discussion}

CyA is the cornerstone of modern immunosuppressive therapy despite its well-known side effects. Jeanmart and colleagues $^{33}$ demonstrated that CyA results in impairment of endothelial-dependent vasorelaxation in an in vitro model. Sudhir and colleagues ${ }^{17}$ and Khalil and colleagues ${ }^{34}$ have both shown in dogs that acute exposure to CyA impairs coronary endothelial function. Their results also indicate that NO dysregulation may be involved in CyA-induced dysfunction. ${ }^{17,34}$ Our study confirms that both $\mathrm{CyA}$ and Hcort result in vasomotor dysfunction in an in vivo model. The mechanisms of injury are different between the 2 agents, and when they were administered concomitantly, we observed a synergistic impairment of vascular function. The possible mechanisms of vascular injury include direct oxidant injury, increased cytokine release, and altered regulation of endothelial NO production.

We demonstrated that CyA treatment significantly alters normal vascular homeostasis as assessed by impaired endothelial-dependent vascular dilatation. Normal vessel function is maintained by the balance between NO and ET-1. Our study revealed that CyA alters both $\mathrm{NO}$ and $\mathrm{ET}-1$ regulation. First, eNOS protein expression was reduced after treatment with CyA, indicating a possible impairment in NO regulation. The reduction in eNOS protein expression may be
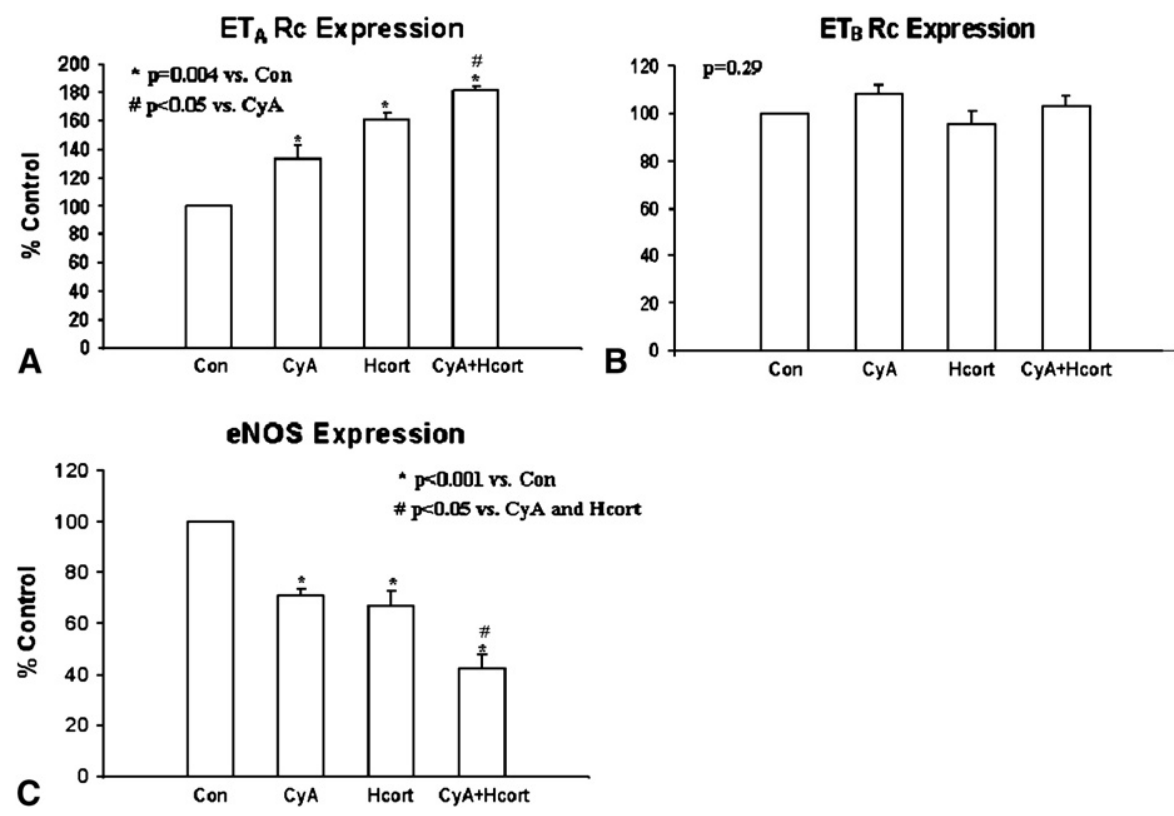

Figure 2. A, Quantitative Western blot analysis of $\mathrm{ET}_{A} \mathrm{Rc}$ expression in the TAo. All treatment groups showed increased $\mathrm{ET}_{\mathrm{A}} \mathrm{Rc}$ expression, with the greatest increase observed in the CyA + Hcort group. B, Quantitative Western blot analysis of $\mathrm{ET}_{B}$ Rc protein expression in the TAo. No differences were observed between groups. C, Quantitative Western blot analysis of eNOS protein expression in the TAo. Both CyA and Hcort decreased eNOS expression, with CyA + Hcort demonstrating a synergistic reduction. $E T$, Endothelin; $R c$, receptor; Con, control; $C y A$, cyclosporine $A$; Hcort, hydrocortisone; eNOS, endothelial nitric oxide synthase. 
a consequence of CyA inhibiting cyclophilin cis-trans peptidyl-prolyl isomerase function, resulting in impaired eNOS folding and therefore increased degradation. Lungu and colleagues $^{35}$ showed that CyA also inhibits eNOS activity. Second, although ET-1 levels were not elevated by CyA treatment, $\mathrm{ET}_{\mathrm{A}} \mathrm{Rc}$ protein expression in the TAo was significantly increased with no concomitant change in $\mathrm{ET}_{\mathrm{B}} \mathrm{Rc}$ protein expression. $\mathrm{ET}_{\mathrm{A}} \mathrm{Rc}$ activation results in vasoconstriction, whereas $\mathrm{ET}_{\mathrm{B}} \mathrm{Rc}$ on endothelial cells results in vasodilation. Therefore, an increased $\mathrm{ET}_{\mathrm{A}}$ Rc-to-ET $\mathrm{ET}_{\mathrm{B}} \mathrm{Rc}$ ratio results in greater vasoconstriction. Thus, CyA results in greater sensitivity to ET-1-induced vasospasm. Hunley and colleagues ${ }^{36}$ showed in a rodent model that CyA-induced renal dysfunction was abrogated after treatment with $\mathrm{BQ123}$, an $\mathrm{ET}_{\mathrm{A}} \mathrm{Rc}$ antagonist. Takeda and colleagues ${ }^{37}$ observed a similar finding in mesangial cells where ET-1 antagonism protected against CyA-induced injury. Therefore, $\mathrm{ET}_{\mathrm{A}} \mathrm{Rc}$ up-regulation may be a common pathway by which CyA results in injury in various cell types. CyA also resulted in a higher SNP $\mathrm{ED}_{50}$ compared with the other treatment groups, indicating impaired cyclic guanosine monophosphate-dependent smooth muscle cell relaxation. The study by Khalil and colleagues ${ }^{34}$ reinforces our observations. They demonstrated that CyA impaired both endothelial-dependent and independent vasodilation. Previous studies by Diederich and colleagues $^{29}$ and Galle ${ }^{38}$ have suggested that CyA treatment increases free radical production. In comparison with our investigations, the study by Diederich and colleagues ${ }^{29}$ used an elevated dose of CyA $(25 \mathrm{mg} / \mathrm{kg})$, and the study by Galle and colleagues ${ }^{38}$ was performed on isolated vascular rings and not in an in vivo model. Our study confirmed that oxidative injury (increased 8-isoprostane levels) occurred after even low-normal CyA exposure in an in vivo model. Oxidative injury may also account for the smooth muscle dysfunction observed after CyA therapy. In addition, oxygen radicals can inhibit NO production, worsening vascular homeostasis. Proinflammatory cytokines can aggravate oxidative injury and represent a possible mechanism of injury after either CyA or Hcort exposure. However, we found no significant changes in myocardial levels of either transforming growth factor- $\alpha$ or tumor necrosis factor- $\beta$, suggesting that a heightened inflammatory response is not the cause of our observed derangements. Additional markers of inflammation need to be assessed to confirm this conclusion.

Corticosteroids are also associated with impaired vasomotor function. Unlike CyA, Hcort did not impair endothelialdependent vascular dilatation. However, Hcort did result in a similar decrease in eNOS protein expression, indicating that impaired NO regulation seen with CyA may be due to impaired enzyme function in addition to decreased protein expression. There was no impairment in endothelial-independent vasodilation with Hcort therapy. Hcort treatment did result in increased sensitivity to ET-1. Hcort exposure increased $\mathrm{ET}_{\mathrm{A}} \mathrm{Rc}$ expression with no change in $\mathrm{ET}_{\mathrm{B}} \mathrm{Rc}$ expression. Unlike CyA, Hcort treatment did not result in oxidative injury. Thus, we speculate that CyA treatment results in functional uncoupling of the eNOS enzyme producing free radicals instead of NO. Furthermore, functional uncoupling of eNOS by CyA may explain why similar reductions in protein expression did not result in impaired endothelial vasodilation with Hcort. Transplant recipients are commonly given both a corticosteroid and CyA. We demonstrated that Hcort therapy aggravates CyA-induced smooth muscle dysfunction as indicated by worsened endothelialindependent vasorelaxation and heightened sensitivity to ET-1 vasospasm.

We have made the following novel observations (Table 1):

1. CyA results in alteration of both NO and ET-1 regulation, leading to impairment of vasodilation and increased sensitivity to vasospasm.

2. Hcort results in decreased eNOS expression and increased $\mathrm{ET}_{\mathrm{A}} \mathrm{Rc}$ expression, resulting in increased sensitivity to vasospasm.

3. CyA results in direct oxidative injury to the endothelium.

4. Corticosteroid treatment aggravates CyA-induced vasomotor dysfunction.

Our findings suggest possible treatment strategies for improving vasomotor function in patients receiving standard immunosuppression. An effective strategy must include

TABLE 1. Summary of vascular effects of cyclosporine A, hydrocortisone, and cyclosporine + hydrocortisone

\begin{tabular}{llccc}
\hline & & CyA & Hcort & CyA + Hcort \\
\hline Vasoreactivity to ET-1 & \%Cmax & $256 \% \pm 11 \%^{*}$ & $256 \% \pm 11 \%^{*}$ & $348 \% \pm 10 \%^{*} \dagger$ \\
& ET-1 levels & $0.98 \pm 0.1 \mathrm{fmol} / \mathrm{L}$ & $0.97 \pm 0.1 \mathrm{fmol} / \mathrm{L}$ & $1.0 . \pm 0.1 \mathrm{fmol} / \mathrm{L}$ \\
& ROS & $150 \% \pm 6 \%^{*}$ & $113 \% \pm 2 \%$ & $162 \% \pm 9 \%^{*}$ \\
& ET-1 Rc A & $133 \% \pm 10 \%^{*}$ & $161 \% \pm 5 \% \%^{*}$ & $181 \% \pm 3 \%^{*} \dagger$ \\
Vasoreactivity to acetylcholine & ET-1 Rc B & $108 \% \pm 4 \%$ & $96 \% \pm 5 \%$ & $103 \% \pm 4 \%^{*}$ \\
& $\% E m a x$ & $33 \% \pm 2 \%^{*}$ & $51 \% \pm 1 \%$ & $30 \% \pm 7 \%^{*}$ \\
& eNOS & $71 \% \pm 3 \%^{*}$ & $67 \% \pm 6 \% \%^{*}$ & $43 \% \pm 5 \%^{*} \dagger$ \\
& ROS & $150 \% \pm 6 \%^{*}$ & $113 \% \pm 2 \%$ & $162 \% \pm 9 \%^{*}$ \\
\hline
\end{tabular}

$R c$, Receptor; $E T$, endothelin; ROS, reactive oxygen species; eNOS, endothelial nitric oxide synthase. ${ }^{*} P<.05$ versus control. $\dagger P<.05$ versus CyA and Hcort. 
ET-1 antagonism in addition to functional coupling of eNOS. This may be achieved by using an ET-1 antagonist (eg, bosentan) and tetrahydrobiopterin, an essential eNOS cofactor, for stabilizing the eNOS complex and reducing free radical production. In the case of corticosteroids, an effective strategy must include ET-1 antagonism and an NO donor. This may be achieved by using bosentan and L-arginine, an NO donor, to counterbalance the increased sensitivity to ET-1. Our findings provide potential mechanisms for the development of CyA and corticosteroid-induced hypertension, as well as a direct mechanism by which CyA may lead to transplant vasculopathy. Further studies will delineate the putative beneficial effects of our proposed treatment strategies.

\section{Conclusions}

The present study was designed to investigate the direct effect of our treatment groups on vascular function in the absence of an immune response or a period of ischemia/reperfusion. Clearly, the effects of the latter 2 variables will need further assessment in a heterotopic transplant model. In addition, we evaluated changes in aortic tissue as opposed to coronary arteries. Although the responses are likely consistent, it is possible that both CyA and Hcort exert differential effects on coronary vasculature than seen in the TAo.

\section{References}

1. Billingham ME. Cardiac transplant atherosclerosis. Transplant Proc. 1987;19(4 Suppl. 5):19-25.

2. Kahan BD. Cyclosporine. N Engl J Med. 1989;321:1725-38.

3. Paul LC. Overview of side effects of immunosuppressive therapy. Transplant Proc. 2001;33:2089-91.

4. Buetler TM, Cottet-Maire F, Krauskopf A, Ruegg UT. Does cyclosporin A generate free radicals? Trends Pharmacol Sci. 2000;21:288-90.

5. Calne RY, Collier DS, Lim S, Pollard SG, Samaan A, White DJ, et al. Rapamycin for immunosuppression in organ allografting. Lancet. 1989;2:227.

6. Meiser BM, Billingham ME, Morris RE. Effects of cyclosporin, FK506, and rapamycin on graft-vessel disease. Lancet. 1991;338:1297-8.

7. Morris ST, McMurray JJ, Rodger RS, Farmer R, Jardine AG. Endothelial dysfunction in renal transplant recipients maintained on cyclosporine. Kidney Int. 2000;57:1100-6.

8. Paul LC. Endothelial dysfunction, atherosclerosis and cyclosporine in renal transplantation. Neth J Med. 2001;59:1-3.

9. Schrama YC, van Dam T, Fijnheer R, Hene RJ, de Groot P, Rabelink TJ. Cyclosporine is associated with endothelial dysfunction but not with platelet activation in renal transplantation. Neth J Med. 2001;59:6-15.

10. Zoja C, Furci L, Ghilardi F, Zilio P, Benigni A, Remuzzi G. Cyclosporin-induced endothelial cell injury. Lab Invest. 1986;55:455-62.

11. Drueke TB, Abdulmassih Z, Lacour B, Bader C, Chevalier A, Kreis H. Atherosclerosis and lipid disorders after renal transplantation. Kidney Int Suppl. 1991;31:S24-8.

12. Kronenberg F, Konig P, Lhotta K, Konigsrainer A, Sandholzer C, Utermann $\mathrm{G}$, et al. Cyclosporin and serum lipids in renal transplant recipients. Lancet. 1993;341:765.

13. Lau DC, Wong KL, Hwang WS. Cyclosporine toxicity on cultured rat microvascular endothelial cells. Kidney Int. 1989;35:604-13.

14. Bossaller C, Forstermann U, Hertel R, Olbricht C, Reschke V, Fleck E. Cyclosporin A inhibits endothelium-dependent vasodilatation and vascular prostacyclin production. Eur J Pharmacol. 1989;165:165-9.

15. Diederich D, Yang Z, Luscher TF. Chronic cyclosporine therapy impairs endothelium-dependent relaxation in the renal artery of the rat. $J$ Am Soc Nephrol. 1992;2:1291-7.
16. Dinh Xuan AT, Fan TP, Higenbottam TW, Wallwork J. Cyclosporine in vitro reduces endothelium-dependent relaxation to acetylcholine but does not affect relaxation to nitrovasodilators. Transplant Proc. 1990; 22:1723-5.

17. Sudhir K, MacGregor JS, DeMarco T, De Groot CJ, Taylor RN, Chou TM, et al. Cyclosporine impairs release of endothelium-derived relaxing factors in epicardial and resistance coronary arteries. Circulation. 1994;90:3018-23.

18. Abraham JS, Bentley FR, Garrison RN. The role of intrarenal prostaglandins and angiotensin II in acute cyclosporine-induced vasoconstriction. Surgery. 1991;110:343-9.

19. Kon V, Sugiura M, Inagami T, Harvie BR, Ichikawa I, Hoover RL. Role of endothelin in cyclosporine-induced glomerular dysfunction. Kidney Int. 1990;37:1487-91.

20. Morgan BJ, Lyson T, Scherrer U, Victor RG. Cyclosporine causes sympathetically mediated elevations in arterial pressure in rats. Hypertension. 1991;18:458-66.

21. Abassi ZA, Pieruzzi F, Nakhoul F, Keiser HR. Effects of cyclosporin A on the synthesis, excretion, and metabolism of endothelin in the rat. Hypertension. 1996;27:1140-8.

22. Bunchman TE, Brookshire CA. Cyclosporine-induced synthesis of endothelin by cultured human endothelial cells. J Clin Invest. 1991;88: 310-4.

23. Carrier M, Tronc F, Stewart D, Pelletier LC. Dose-dependent effect of cyclosporin on renal arterial resistance in dogs. Am J Physiol. 1991; 261(6 Pt 2):H1791-6.

24. Edwards BS, Hunt SA, Fowler MB, Valantine HA, Anderson LM, Lerman A. Effect of cyclosporine on plasma endothelin levels in humans after cardiac transplantation. Am J Cardiol. 1991;67:782-4.

25. Forslund T, Hannonen P, Reitamo S, Fyhrquist F. Hypertension in cyclosporin A-treated patients is independent of circulating endothelin levels. J Intern Med. 1995;238:71-5.

26. Stroes ES, Luscher TF, de Groot FG, Koomans HA, Rabelink TJ. Cyclosporin A increases nitric oxide activity in vivo. Hypertension. 1997;29:570-5.

27. Takeda Y, Itoh Y, Yoneda T, Miyamori I, Takeda R. Cyclosporine A induces endothelin-1 release from cultured rat vascular smooth muscle cells. Eur J Pharmacol. 1993;233:299-301.

28. Calo L, Davis PA, Rigotti P, Milani M, Cantaro S, Marchini F, et al. ecNOS overexpression in CsA-treated renal transplant patients: implications for CsA-induced hypertension. Transplant Proc. 1998;30:2012-3.

29. Diederich D, Skopec J, Diederich A, Dai FX. Cyclosporine produces endothelial dysfunction by increased production of superoxide. Hypertension. 1994;23:957-61.

30. Mehlhorn U, Krahwinkel A, Geissler HJ, LaRosee K, Fischer UM, Klass $\mathrm{O}$, et al. Nitrotyrosine and 8-isoprostane formation indicate free radical-mediated injury in hearts of patients subjected to cardioplegia. J Thorac Cardiovasc Surg. 2003;125:178-83.

31. Waugh RJ, Morrow JD, Roberts LJ, Murphy RC. Identification and relative quantitation of $\mathrm{F} 2$-isoprostane regioisomers formed in vivo in the rat. Free Radic Biol Med. 1997;23:943-54.

32. Bradford MM. A rapid and sensitive method for the quantitation of microgram quantities of protein utilizing the principle of protein-dye binding. Anal Biochem. 1976;72:248-54.

33. Jeanmart H, Malo O, Carrier M, Nickner C, Desjardins N, Perrault LP. Comparative study of cyclosporine and tacrolimus vs newer immunosuppressants mycophenolate mofetil and rapamycin on coronary endothelial function. J Heart Lung Transplant. 2002;21:990-8.

34. Khalil A, Carrier M, Latour JG, Pelletier LC. Cyclosporin A-induced coronary artery vasoconstriction through myogenic and endotheliumdependent mechanisms. Circulation. 1996;94(9 Suppl):II308-11.

35. Lungu AO, Jin ZG, Yamawaki H, Tanimoto T, Wong C, Berk BC. Cyclosporin A inhibits flow-mediated activation of endothelial nitricoxide synthase by altering cholesterol content in caveolae. J Biol Chem. 2004;279:48794-800.

36. Hunley TE, Fogo A, Iwasaki S, Kon V. Endothelin A receptor mediates functional but not structural damage in chronic cyclosporine nephrotoxicity. J Am Soc Nephrol. 1995;5:1718-23.

37. Takeda M, Breyer MD, Noland TD, Homma T, Hoover RL, Inagami T, et al. Endothelin-1 receptor antagonist: effects on endothelin- and cyclosporine-treated mesangial cells. Kidney Int. 1992;41:1713-9. 
38. Galle J, Lehmann-Bodem C, Hübner U, Heinloth A, Wanner C. CyA and OxLDL cause endothelial dysfunction in isolated arteries through endothelin-mediated stimulation of $\mathrm{O}(2)(-)$ formation. Nephrol Dial Transplant. 2000;15:339-46.

\section{Discussion}

Dr Frank Sellke (Boston, Mass). I noticed for several of the parameters you looked at, in particular endothelial-dependent relaxation, Hcort itself really didn't have any effect, but when you added it to CyA, it seemed to aggravate the detrimental effects. Why do you see this added detrimental action of Hcort when by itself doesn't cause any impaired effect?

Dr Ramzy. When we looked at endothelial-dependent vasorelaxation, we did not see a significant difference. There are 2 sides to NO production: There is the expression of eNOS and the activity of eNOS. Because of time constraints I did not show all our data. CyA has direct inhibitory effects on eNOS in addition to its ability to decrease its expression, whereas Hcort demonstrated decreased expression with no change in activity. Thus, when we looked at endothelial-dependent vasorelaxation, we did not find any significant differences with Hcort. However, when we looked at Hcort's effect on sensitivity to ET-1, there was significantly aggravated ET-1-induced vasospasm because it has the ability to up-regulate the ETA receptor, which is made even worse when we add CyA. When we combined the 2 , the expression of eNOS is significantly decreased compared with CyA alone. With the added effect of CyA directly inhibiting eNOS activity, we end up with worsening endothelial dysfunction.

Dr Chadwick Stouffer (Grand Rapids, Mich). In your 4 groups, it looked like your ET-1 levels were all comparable. Can you comment on your conclusion that that did affect homeostasis? How did you look at the oxygen-free radical production in the CyA group?

Dr Ramzy. When we looked at the figure for ET-1 production, the levels were the same in our 3 main groups. However, in a situation of decreased NO production (again, because of the time con- straint I did not demonstrate these data), our 3 main groups impaired NO production. Under normal conditions with an inhibition of NO production, ET-1 levels should decrease. Therefore, maintaining ET-1 levels at control levels is actually a relative increase in ET-1; therefore, at those levels it was a relative elevation of ET-1 compared with our control group. We measured ET-1 levels at the same time we determined our trough levels for CyA, and several studies have demonstrated that CyA on its own increases ET-1 levels in a concentration-dependent manner. As CyA levels change, ET-1 production changes. Therefore, if we measure ET-1 levels at peak concentration, I suspect we will see higher levels. Dr Takeda's group demonstrated that 6 weeks of CyA treatment elevated levels of ET-1.

In addition to our data demonstrating free-radical injury, we also looked at a cell culture model whether these drugs are able to produce free radicals, and CyA, as seen here, has the ability to increase free-radical production compared with control.

Dr Mark Krasna (Towson, Md). Dr Ramzy, perhaps you could just elaborate a little bit then on the possible modifications in treatment strategies that you would use now?

Dr Ramzy. We actually performed additional studies looking at whether our theories would actually work. Tetrahydrobiopterin (BH4) is an essential cofactor for eNOS, and when we give this to our CyA treatment group we do see a restoration to near normal of vasomotor function in terms of endothelial-dependent vasorelaxation with $\mathrm{BH} 4$, demonstrating the importance of eNOS dimerization. Therefore, CyA not only decreases eNOS expression but also results in uncoupling of the enzyme, which produces more free radicals than NO. The addition of Bosentan, an ET-1 antagonist, completely restores endothelial-dependent vasorelaxation, and similarly with endothelial-independent vasorelaxation, $\mathrm{BH} 4$ has the ability to attenuate CyA effects. As you can see in this figure, there is still a lag, indicating the NO side of the equation is not the only player, that there is, in addition to NO impairment, another factor, which our data suggest is ET-1. Thus, we believe that optimal therapy should consist of both eNOS augmentation and ET-1 blockade. 